\title{
Does Risk Aversion Affect Transmission and Generation Planning? A Western North America Case Study
}

\author{
Francisco D. Munoz ${ }^{\mathrm{a}, *}$, Adriaan Hendrik van der Weijde ${ }^{\mathrm{b}}$, Benjamin F. \\ Hobbs $^{\mathrm{c}}$, Jean-Paul Watson ${ }^{\mathrm{d}}$ \\ ${ }^{a}$ Facultad de Ingeniería y Ciencias, Universidad Adolfo Ibáñez, Diagonal Las Torres 2640, \\ Peñalolén, Santiago, Chile. \\ ${ }^{b}$ School of Engineering, The University of Edinburgh, The King's Buildings, Mayfield Road, \\ Edinburgh EH9 2JL, UK. \\ ${ }^{c}$ Whiting School of Engineering, Johns Hopkins University, $3400 \mathrm{~N}$ Charles St, Baltimore, \\ MD 21218, USA. \\ ${ }^{d}$ Department of Discrete Math and Optimization, Sandia National Laboratories, P.O. Box \\ 5800, MS 1326, Albuquerque, NM 87185-1326, USA.
}

\begin{abstract}
We investigate the effects of risk aversion on optimal transmission and generation expansion planning in a competitive and complete market. To do so, we formulate a stochastic model that minimizes a weighted average of expected transmission and generation costs and their conditional value at risk $(\mathrm{CVaR})$. We show that the solution of this optimization problem is equivalent to the solution of a perfectly competitive risk-averse Stackelberg equilibrium, in which a risk-averse transmission planner maximizes welfare after which risk-averse generators maximize profits. This model is then applied to a 240-bus representation of the Western Electricity Coordinating Council, in which we examine the impact of risk aversion on levels and spatial patterns of generation and transmission investment. Although the impact of risk aversion remains small at an aggregate level, state-level impacts on generation and transmission investment can be significant, which emphasizes the importance of explicit consideration of risk aversion in planning models.
\end{abstract}

Keywords: risk aversion, stochastic programming, transmission and generation planning, investment

\section{Introduction}

Transmission planners in liberalized electricity markets face large amounts of uncertainty. This includes short-term uncertainty about demand, intermittent generation, and equipment outages, but more importantly, long-term fuel prices, load growth, construction cost, and policy uncertainty. The amount of

${ }^{*}$ Corresponding author. E-mail: fdmunoz@uai.cl

C) 2017. This manuscript version is made available under the Elsevier user license http://www.elsevier.com/open-access/userlicense/1.0/ 
both short-term and long-term uncertainty is likely to increase even further in the coming decade, with increasing amounts of renewable generation capacity, increasing uncertainty about the availability of fossil fuels, and worldwide proliferation of policies to stimulate renewable development. This has implications for investment, since investments in both transmission and generation capacity usually have very long lead times of multiple years or even decades, and decisions are not easily reversible (Fuss et al., 2008; Barradale, 2010; Hu and Hobbs, 2010).

To allow transmission planners to make better decisions in this uncertain environment, stochastic planning models have been developed (see, e.g., De la Torre et al. 1999; Sauma and Oren 2006; Roh et al. 2009; van der Weijde and Hobbs 2012; Munoz et al. 2014; Go et al. 2016). However, these models usually assume risk-neutral transmission planners, and that generation firms that invest in new capacity following transmission are, likewise, risk neutral. Most empirical evidence on investments suggests that decision makers, whether public or private, are instead risk averse. ${ }^{1}$ Modeling of risk aversion might change near-term investments, for instance by increasing the attractiveness of delaying investments in order to gain more information, or by increasing the value of diverse portfolios of transmission investments that avoid the risk of poor performance under some future scenarios. Risk neutral stochastic transmission planning models may therefore a) be inappropriate if the transmission planner is risk averse and b) incorrectly model the response of risk averse generators to transmission investment.

Others have analyzed the impact of risk aversion, and therefore the effect of a simplifying risk-neutrality assumption, on transmission and generation planning problems; some of this literature is surveyed in Section 2 below. However, the vast majority of these studies only look at either generation or transmission investment, and fail to capture the important interactions between the two that have been identified in the earlier transmission-generation planning literature (e.g., Munoz et al., 2014; van der Weijde and Hobbs, 2012). Moreover, they are generally based on very small models, which are not necessarily representative of large real-world transmission networks and cannot capture the full spatial patterns of transmission and generation investment.

This paper is a first attempt to investigate the impact of risk aversion on the results of large-scale electricity planning models that represent the interactions between transmission and generation investment. We compare the transmission and generation expansion plans identified by such a model under assumptions of risk neutrality and risk aversion, to see where risk aversion makes a difference, and consequently, whether the existing studies and models that assume risk

\footnotetext{
${ }^{1}$ As discussed in Munoz et al. (2015), both the Midcontinent and the California Independent System Operators use engineering rules that aim at identifying "robust" or "least regret" transmission projects. Although risk aversion is not explicitly mentioned in these studies, their methodologies suggest that the planning authorities are more concerned with worst-case situations (i.e., risk averse preferences) than with the expected performance of the selected projects across all considered scenarios (i.e., risk neutrality).
} 
neutrality are adequate or not.

We model a proactive risk-averse transmission planner, who maximizes a risk-adjusted measure for social welfare, and, because transmission expansion changes nodal electricity prices, anticipates a response by risk averse investors in generation capacity. As we will see, the solution to this Stackelberg equilibrium problem is, under some reasonable assumptions, equivalent to a risk-averse cost minimization, allowing us to solve the problem at scale for a 240-bus representation of the Western Electricity Coordinating Council (WECC) network of North America.

Naturally, our approach has limitations: we only model a single decision stage, the complex interactions between individual generators and between generators and the transmission planner that occur in real-world imperfectly competitive markets are not fully captured, and we use a simple case study with a linearized DC representation of the electrical flows on the network. Nevertheless, our results do indicate that risk aversion can have impact on the amount of investment in transmission and generation capacity, on the type of capacity, and on the spatial distribution of that capacity.

The next section will review some of the existing literature on risk-averse generation and transmission planning. In Section 3 we describe our methodology and derive the equivalence of the risk-averse Stackelberg equilibrium problem and the risk-averse cost minimization. Section 4 summarizes the assumptions and approach of the WECC case study, the results of which follow in Section 5. Section 6 concludes.

\section{Existing literature}

In this section we first overview different methods to include risk aversion in planning models. We then briefly review the existing literature on risk-averse generation and transmission planning.

\subsection{Modeling risk aversion}

There are several ways to include risk aversion in planning models. In the economics literature, concave utility functions are popular: these can be used to convert monetary costs (or profits) into utilities, whose expected value is then optimized instead of the original objective (Fishburn, 1970). Possible specifications for the utility functions include exponential functions (exhibiting constant absolute risk aversion, CARA) and isoelastic functions (exhibiting constant relative risk aversion, CRRA) (Eeckhoudt et al., 2005). These functions are, of course, non-linear, which makes including them in large-scale planning models challenging. If, in addition to investors being CARA risk averse, the distribution of possible outcomes is normal, the exponential utility function can be written as a linear combination of expected outcomes and the standard deviation of the outcome distribution, which is quadratic. This mean-variance utility approach simplifies the problem significantly, which is one of the reasons for its popu-

larity, but it is, unfortunately, often used in settings where the assumption of 
normality is clearly invalid (such as a stochastic planning problem with a small number of scenarios).

Another way to model risk aversion, which originates in the financial mathematics literature, is to include the value at risk (VaR) (Duffie and Pan, 1997) or conditional value at risk (CVaR) (Rockafellar and Uryasev, 2000) in the decision maker's objective or constraints. VaR gives the probability that outcomes are worse than a given threshold; however, its mathematical properties are unattractive. CVaR gives the expected outcome over outcomes that are worse than the VaR. Rockafellar and Uryasev (2000) demonstrate that, for a given quantile, the CVaR can be computed as part of the solution of a simple linear program, which makes its inclusion in large-scale planning models relatively straightforward.

Finally, robust planning models find the minimum cost solution that is feasible under a range of potential realizations of uncertain variables (Mulvey et al., 1995; Ben-Tal and Nemirovski, 2002). A wide range of different formulations has been proposed: some only include constraints that enforce feasibility in all scenarios without considering costs, whereas others are closer to CVaR-based models in that they minimize worst-case costs or maximize worst-case outcomes. The advantage of this approach is that the probabilities of future scenarios do not have to be defined; however, without them, risk aversion is limited to the worst-case outcome and the expected performance of the solution cannot be evaluated.

All of these methods have been applied to transmission and generation planning; Sections 2.2 and 2.3 give an overview of some of these studies and their results, without aiming to be a comprehensive literature review.

\subsection{Risk-averse generation planning}

Several studies have used the above methods to consider the effect of risk aversion on investment in electricity generation capacity, usually in the setting of a perfectly competitive market. Using theoretical economic models, Neuhoff and de Vries (2004) show that if consumers and investors are risk averse, and these risks cannot be traded, competitive markets will not deliver enough investment because risk premia increase generator costs. Moreover, they skew the generation mix towards less risky, less-capital intensive technologies, which is also undesirable from a social perspective, and is a serious barrier to investment in renewables. Ehrenmann and Smeers (2011a,b) show similar effects using stochastic equilibrium models with CVaR-maximizing investors or stochastic discount rates. In their models, which feature uncertain fuel costs, emissions reduction targets, and numbers of carbon allowances, risk averse investors build more open cycle gas turbines and less coal-fired generation capacity. This is because the latter have a higher up-front capital cost and are therefore more risky; however, they also show that there are important interactions between risk aversion and model constraints, such as price caps or carbon targets. Fan et al. (2010), which has investors maximizing utility functions that exhibit constant absolute risk aversion, shows that the way these carbon targets are implemented is highly relevant as well. If a carbon taxed or auctioned permit scheme is anticipated, risk averse generators prefer cleaner generation technologies to ensure 
against these regulatory costs. If, on the other hand, grandfathered permits are considered, this favors investment in carbon-intensive generation capacity.

These papers focus on project-based investment decisions, such that potential investment in each technology is evaluated separately. Roques et al. (2008) take a different approach and use mean-variance optimization to investigate how risk averse investors would choose a portfolio of technologies. They illustrate the importance of correlations between the uncertain variables in this context: if, as is often the case, gas prices and electricity prices are highly correlated, this makes investment in gas-fired generation capacity less risky (as high input price, which decreases profits, will usually coincide with a high electricity price, which compensates for the increase in input prices). Hence, risk averse investors favor gas-fired generation capacity, rather than nuclear or coal-fired. In their model, electricity prices are exogenous. A similar analysis is done in Huang and $\mathrm{Wu}$ (2008) for Taiwan, but focusing on minimizing the risk-weighted value of total generation cost. Meunier (2013) shows similar interactions between technologies in a portfolio-style model with endogenous prices. In this model, investment in peaking capacity may also increase with risk aversion, not because of exogenous correlations but because peaking capacity sets prices, which makes it useful to hedge against the the returns on investment in baseload capacity. The meanvariance approach has also been applied to wind planning. For instance, Roques et al. (2010) use this approach to find a portfolio of cross-country portfolios that minimize the total variance of wind production in Europe. They conclude that additional cross-country transmission capacity and more coordinated renewable energy policies in Europe could significantly increase the efficiency of the wind portfolio. For a general framework on uncertainty and risk analysis in power system planning, we refer to Merrill and Wood (1991).

Most of the models developed in the papers mentioned above are small, with a limited number of scenarios, time periods, and generation technologies. An exception is Jin et al. (2011), which focuses on algorithm design but does show that large problems can still be solved. Moreover, in all of them, transmission constraints are ignored. In Kamalinia et al. (2014), which uses mean-variance optimization to study equilibria in a competitive market, a sensitivity analysis with respect to the transmission constraints shows that, as may be expected, these constraints have a major impact on generator's payoffs, and therefore on investment and the impact of risk aversion. The latter is not quantified, however. This study is also one of the few to explicitly consider investment in renewable generation capacity.

Interestingly, and contrary to what the other studies above seem to suggest, investment in renewables increases with risk aversion, despite their high capital intensity. A similar model proposed by Pisciella et al. (2014), which has investors maximizing a weighted average of expected profits and CVaRs, shows similar results. Driving these results is the fact that renewable generation is not subject to direct fuel price uncertainty, or even demand uncertainty in situations where demand always exceeds renewable generation levels. Its profitability, naturally, depends on market prices, which are still uncertain. However, since input prices are not, investment in renewable generation capacity is, in this model, less risky 
than conventional generation capacity, which is subject to but input and output price uncertainty. Whether this is realistic or not is debatable, but it does illustrate the impact of how uncertain variables and their realizations can have a major impact on how investments are chosen.

Risk aversion has also been considered in the real options literature, where the focus is on the timing of investment. Kettunen et al. (2011) use a multi-stage stochastic optimization model with carbon price uncertainty and constraints on the conditional cash flow at risk (i.e., the $\mathrm{CVaR}$ of the cash flow). Focusing on the temporal aspect of an individual investor only, they neither address technology choice or the system-wide effects of risk aversion; their models also ignore transmission. They do show, however, that it is important to include uncertainties, as well as missing markets and other financial details, because these model features have a large effect in the returns to investment. In a more general real options setting, Hugonnier and Morellec (2007) illustrate the importance of differentiating between variability, which can be hedged and leads investors to postpone decisions, and idiosyncratic risk, which cannot be hedged and may lead to investments being undertaken too early.

\subsection{Risk-averse transmission planning}

Considerably less attention has been given to the effect of risk aversion on optimal transmission expansion plans. Sardinha et al. (2013), using a CVaRbased transmission planning model with uncertain demand, show that higher levels of risk aversion can lead to an increase in the optimal amount of transmission in a simple 6-bus network. Delgado and Claro (2013) examine a similar setting but use multi-objective optimization techniques to analyze transmission investment in three differently configured 3-bus networks. Their results indicate that the sensitivity of transmission investment to risk aversion depends on the network configuration. Neither of these studies consider possibilities for investment in new generation capacity, which seems to at least partially drive their results. Others have used minimization of maximum regret to analyze the effects of uncertain transmission outages on transmission expansion planning in small networks (Alguacil et al., 2010) and large networks (Arroyo et al., 2010). In these studies, an increase in regret aversion increases investment in transmission capacity because this mitigates the costs of an outage, but again, generation investment is not possible.

López et al. (2007) do consider simultaneous generation and transmission expansion, with the planner maximizing a mean-variance utility function (i.e., assuming constant absolute risk aversion, and normally distributed payoffs). In their model, demand, plant availability, and transmission capacity factors are all stochastic. Applications on a 6-bus and 21-bus are discussed, but only in terms of total costs; neither transmission nor generation investments are presented, and no economic analysis is attempted. Similarly, Zheng and Pardalos (2010) analyze simultaneous investment in liquefied natural gas terminals and the gas transmission network in a setting where a planner minimizes costs subject to a CVaR constraint; they also do not present the optimal decisions, or any type of economic analysis of those decisions. 
A range of studies consider robust transmission expansion planning methods. Since these minimize or constrain the cost of the worst possible outcome, they can be considered as modeling extreme cases of risk aversion. Most of these studies conclude that uncertainty and risk aversion increase investment in transmission capacity (for recent examples, see Jabr, 2013; Chen et al., 2014; Ruiz and Conejo, 2015; Moreira et al., 2015). However, these results are at least partly driven by the fact that generation investment is not usually endogenous in robust transmission planning models. If (risk averse) generation planners also respond to uncertainty, it is no longer obvious that an increase in transmission capacity is needed to hedge risks. A transmission planner may, for instance, even reduce transmission investment if generator risk aversion leads to a wider spatial spread of generation capacity.

Finally, we want to highlight the importance of transmission planning models that explicitly consider generation investments either in a vertically integrated setting or in deregulated markets (i.e., generators' response to transmission investments). It has been found that co-optimizing transmission and generation assets simultaneously can yield different transmission investment plans and cost savings of up to $10 \%$ of total system cost compared to generation-only planning in a vertically-integrated setting (Krishnan et al., 2015). ${ }^{2}$ More sophisticated, multi-level equilibrium models have also been proposed to take into account more realistic features of electricity markets. Sauma and Oren (2006), for instance, shows that a proactive transmission planner that takes into account generators' best response to transmission investments can achieve higher social welfare than a reactive planner. In this model, Sauma and Oren (2006) assume that generators make investments and dispatch decisions sequentially, as in a closed-loop model, and that they have market power. This model was latter reformulated as a mixed-integer linear program (Pozo et al., 2013b,a), thus enabling its application to larger and more realistic case studies, but ignoring market power.

\subsection{The role of financial markets}

Most of the literature cited above implicitly or explicitly assumes that risk cannot be traded: investors do not have the option to insure themselves against future states of the world in which their profits are low or negative. In reality, investors can trade in financial markets to hedge many of the risks they are exposed to. Some examples of financial instruments that are used by investors to control their exposure to price risks include financial transmission rights, electricity futures, forwards, swaps, and options (Deng and Oren, 2006). As Willems and Morbee (2010) show, these financial markets have a significant, and usually positive, effect on investment levels.

However, financial markets are not perfect. Not all risk can be traded in financial markets. This includes some systematic risk (e.g. because of timing dis-

\footnotetext{
${ }^{2}$ Note that this equivalent to a competitive market where all generators make both investments and dispatch decisions simultaneously, in an open-loop fashion.
} 
crepancies between financial market and energy markets) and idiosyncratic risk, such as that resulting from policy changes. The most realistic stochastic model would therefore explicitly model a limited number of financial contracts. The difficulties of incorporating this complication in a large multi-level investment model make this very difficult, and to our knowledge it has not been attempted. An alternative is to assume complete financial markets, such that investors can trade all types of risk (e.g., there exist Arrow-Debreu securities for all future scenarios). This reduces the complexity of the problem and, like the assumption of perfect competition in energy markets, can make risk-averse planning problems much more tractable (see, e.g., Ehrenmann and Smeers, 2011b). It may, however result in an overestimation of investment levels and in an underestimation of the strategic interactions between market participants, including the need for individual investors to hold diversified portfolios, although it is unclear what the practical implications of the latter would be for aggregate investment levels.

\section{Methodology}

\subsection{Modeling risk averse equilibria}

As the literature suggests, risk aversion has important implications for investments in generation capacity: the optimal generation mix in a market with risk-averse agents mix is likely to differ from the one where all the agents are risk neutral, although the nature and magnitude of these differences depend on the details of the market in question. Similarly, risk aversion affects optimal transmission expansion plans. Because transmission expansion changes nodal electricity prices and hence, incentives for investment in generation capacity, there are important interactions between transmission and generation expansion planning. The two therefore cannot be considered in isolation, and an analysis of the effects of risk aversion should simultaneously look at both.

The most realistic approach to do this would be to formulate a stochastic bilevel risk-averse model, in which generators and transmission planners maximize a risk-adjusted measure of profits, and different market participants could have different attitudes to risk. However, because such a model is a mathematical problem with equilibrium constraints (MPEC), it is very difficult to solve at the scale required to investigate the effect of risk aversion in real-world networks with thousands of transmission elements and hundreds of generators. Multilevel equilibrium problems are difficult to solve even without risk aversion, and many traditional bi-level stochastic planning models (such as those developed in in van der Weijde and Hobbs (2012) and Munoz et al. (2014)) therefore operate under the assumption that electricity markets are perfectly competitive, which aligns the objectives of the transmission planner and investors in generation capacity, such that the problem can be collapsed to a single cost minimization. This simplification does not in general follow through to risk-averse settings, but it can be extended to include some special cases of risk aversion, under some relatively straightforward assumptions. 
First, we assume that generators maximize a convex combination of expected profits and the CVaR of the lower tail of these profits and that, as in the models above, generation investment and generation dispatch levels are continuous variables and have constant marginal costs ${ }^{3}$ to ensure that each generator solves a linear profit maximization problem. Second, we assume that the electricity market is perfectly competitive such that each generator is a price taker. ${ }^{4}$ In addition, we now also implicitly assume that a complete financial market exists (i.e., all market participants can trade in financial products that cover every possible future scenario), although we do not model its equilibrium explicitly. As mentioned above, this is an obvious simplification since modeling of financial markets is outside the scope of this work, but it would be even less appropriate to ignore their (beneficial) effects altogether. It has been shown that, given these assumptions, the generation dispatch and generation expansion equilibrium is equivalent to the solution of a risk-adjusted cost minimization problem (Ralph and Smeers, 2010; Ehrenmann and Smeers, 2011b) ${ }^{5}$. This is intuitive - complete financial markets ensure that the worst-case scenario is the worst case for every market participant; perfect competition then restores the market equilibrium to its risk-averse social optimum.

Finally, we assume that the transmission planner acts as a Stackelberg leader who maximizes a convex combination of expected social welfare and the CVaR of the lower tail of the social welfare distribution and use the same weights and CVaR thresholds as the generators. Given that we already know that the generator's equilibrium is equivalent to the outcome of a risk-averse cost minimization, it is then straightforward to prove that solution to the whole bilevel transmission-generation problem can also be found through a risk-averse cost minimization. To see this, we can write the full bi-level problem as follows:

$$
\max _{x} f(x)+g(y)
$$

s.t.

$$
\begin{gathered}
F(x) \leq 0 \\
y=\arg \max _{y} g(y) \quad \text { s.t. } G(x, y) \leq 0
\end{gathered}
$$

The function $f(x)$ is the (risk-averse) cost of transmission expansion, $g(y)$ the (risk-averse) cost of generation and $G(x, y)$ a set of constraints on generation that link the two. The reduced problem is simply the following one:

\footnotetext{
${ }^{3}$ It is possible to use piecewise linear cost functions to approximate increasing marginal costs

${ }^{4}$ More general assumptions are possible, for an example see Sauma and Oren (2006) and Pozo et al. (2013b).

${ }^{5}$ e.g., if all market participants minimize a weighted average of expected costs and the CVaR of the lower tail of the cost distribution, the equivalent single optimization problem minimizes the sum of the individual participants' objectives
} 


$$
\max _{x, y} f(x)+g(y)
$$

s.t.

$$
\begin{gathered}
F(x) \leq 0 \\
G(x, y) \leq 0
\end{gathered}
$$

Assume we have found a solution $\left\{x^{*}, y^{*}\right\}$ to the reduced problem. Then, by Bellman's Principle of Optimality (Bellman, 1952), given $x^{*}, y^{*}$ must satisfy Eq. 3, and hence, the two problems are equivalent. Hence, as in the risk-neutral case, we can collapse the bi-level equilibrium problem to a (linear) optimization problem, as long as the assumptions outlined above are met.

\subsection{Model structure}

To introduce the model we first describe the nomenclature. With the exception of the parameters $\alpha, \Phi$, and $\omega$, we use capitalized letters for all sets and parameters.

Sets and indices

$B \quad$ set of buses, indexed $b$

$B_{p} \quad$ subset of buses in state (subregion) $p$

$B_{j} \quad$ subset of buses in control area $j$

$H$ set of representative dispatch periods, indexed $h$

$G \quad$ set of generators, indexed $i$

$G_{b} \quad$ subset of generators at bus $b$

$G_{j} \quad$ subset of generators in control area $j$

$G_{p} \quad$ subset of generators in state $p$

$G_{R} \quad$ subset of renewable generators

$G_{C}$ subset of candidate generators

$G_{E} \quad$ subset of existing generators

$G_{N} \quad$ subset of intermittent generators

$N \quad$ set of intermittent generators, indexed $n$

$J \quad$ set of control areas, indexed $j$

$L \quad$ set of transmission lines, indexed $l$

$L_{b} \quad$ subset of lines connected to bus $b$

$P \quad$ set of states, indexed $p$

$S \quad$ set of scenarios, indexed $s$ 
Parameters

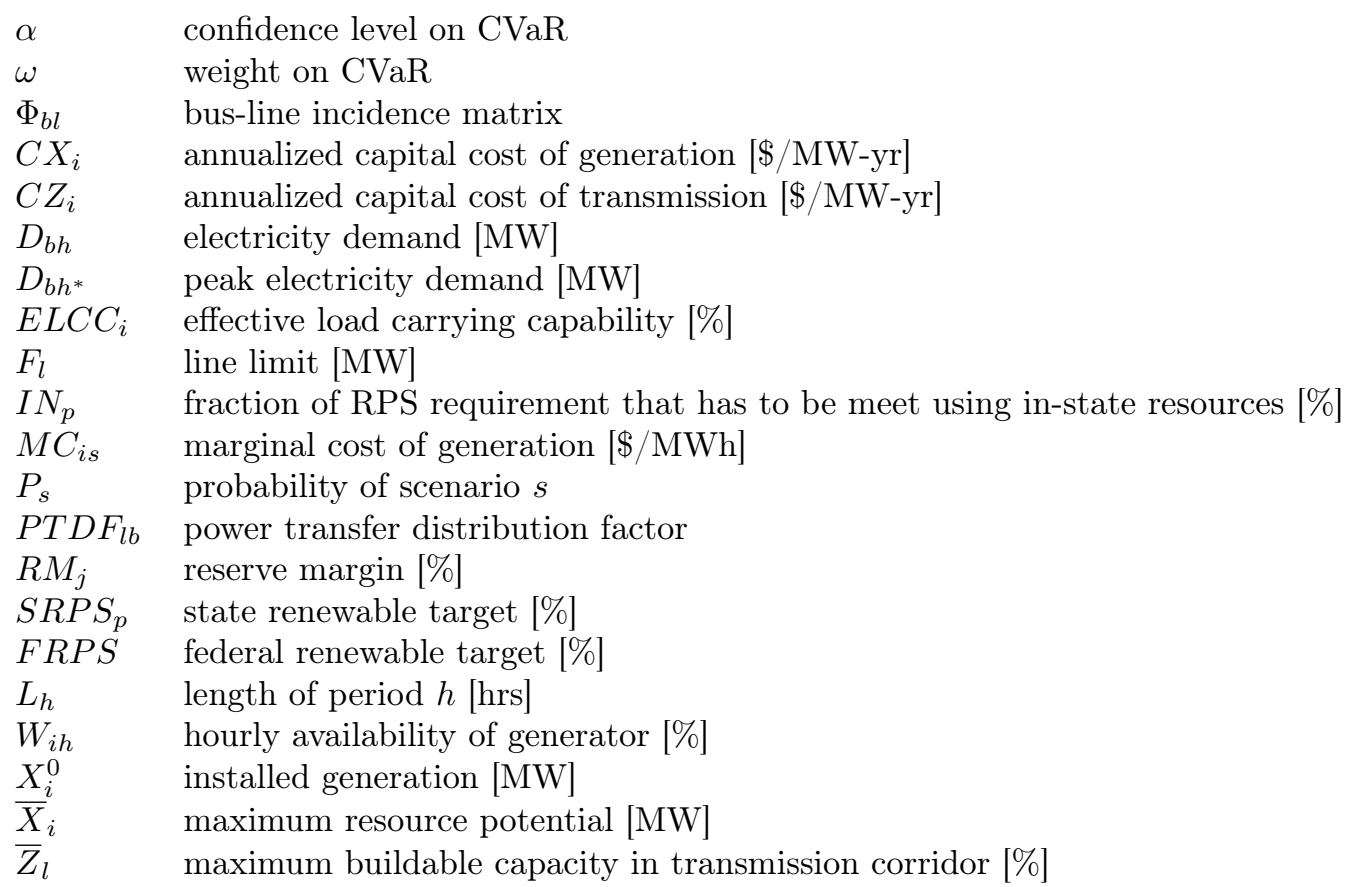

Decision variables

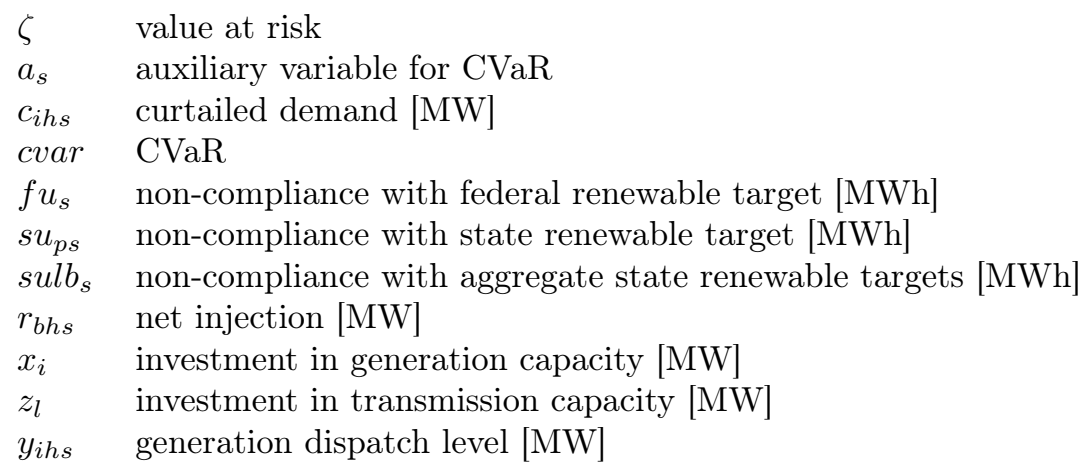

We formulate the investment-planning problem as a two-stage stochastic program. The first-stage decision variables correspond to generation and transmission investment levels, denoted $x_{i}$ and $z_{l}$, respectively. The expression $C C$ corresponds to sum of the annualized investment costs, which we define as follows:

$$
C C=\sum_{i \in G} C X_{i} x_{i}+\sum_{l \in L} C Z_{l} z_{l}
$$


In the second stage there are economic dispatch decisions for dispatch period in each scenario $s$ of economic, market, and regulatory conditions. We denote $O C_{s}$ the operating cost of the system for a representative year in the future.

$O C_{s}=\sum_{h \in H} L_{h}\left(\sum_{i \in G} M C_{i s} y_{i h s}+\sum_{b \in B} V O L L c_{b h s}\right)+N C\left(f u_{s}+s u l b_{s}+\sum_{p \in P} s u_{p s}\right)$

Note that the expression $O C_{s}$ also accounts for the opportunity costs of curtailed demand through the Value of Lost Load (VOLL) and non-compliance with state and federal renewable targets through non-compliance fines $(N C)$, which are assumed to be equal for all federal and state RPS systems. Given these expressions, we define the objective function of the optimization problem as follows:

$$
\min C C+(1-\omega) \sum_{s \in S} P_{s} O C_{s}+\omega \mathrm{cvar}
$$

For $\omega=0$, the problem minimizes the sum of capital cost plus the expect value of operating costs across all scenarios $s \in S$. For $\omega=1$, the objective of the planning problem becomes the minimization of the Conditional Value at Risk, the expectation of the cost over the most costly $\alpha \%$ of scenarios only ${ }^{6}$, denoted with the auxiliary variable cvar. For $0<\omega<1$, the problem effectively puts a higher weight on the most costly $\alpha \%$ of scenarios. Note that since the annualized investment costs $C C$ only depend on first-stage variables - which are not dependent on the second-stage scenarios - this term is not weighed by the parameter $\omega$ and it is outside of the cvar expression.

The co-optimization of transmission and generation investments is done subject to a series of constraints that we describe as follows:

CVaR constraints: We use the linearized formulation of the Conditional Value at Risk proposed by Rockafellar and Uryasev (2000) as follows:

$$
\begin{aligned}
& \zeta+\frac{1}{1-\alpha} \sum_{s \in S} P_{s} a_{s} \leq c v a r \\
& a_{s} \geq O C_{s}-\zeta
\end{aligned}
$$

Market clearing constraints: The difference between generation and demand equals a net injection at every bus in the system.

$$
\sum_{i \in G_{b}} y_{i h s}+c_{b h s}-D_{b h s}=r_{b h s} \quad \forall b \in B, \forall h \in H, \forall s \in S
$$

Generation limits: We assume that all generators are dispatchable. To model the intermittency of renewable resources, including hydro, wind, and solar, we

\footnotetext{
${ }^{6}$ Note that 'most costly' is endogenously defined - the model will determine which scenarios are the most costly considering that decisions have been optimized
} 
limit the dispatch of these technologies to a fraction of their nameplate capacity through hourly availability factors $\left(W_{i h}\right)$. For all non-intermittent generation technologies, the parameters $W_{i h}$ are equal to $1 .^{7}$

$$
y_{i h s} \leq W_{i h}\left(X_{i}^{0}+x_{i}\right) \quad \forall i \in G, \forall h \in H, \forall s \in S
$$

Transmission limits: Since we define net injections per bus, we enforce both thermal limits and Kirchhoff's Voltage Law simultaneously using Power Transfer Distribution Factors (PTDFs) through a single set of constraints. ${ }^{8}$

$$
T_{l} z_{l} \leq \sum_{b \in B} P T D F_{l b} r_{b h s} \leq T_{l} z_{l} \quad \forall l \in L, \forall h \in H, \forall s \in S
$$

Net injections:

$$
\sum_{b \in B} r_{b h s}=0
$$$$
\forall b \in B, \forall h \in H, \forall s \in S
$$

RPS constraints: We enforce one federal renewable target for all eligible states (14) and individual renewable targets per state, as in Short et al. (2011) and Munoz et al. (2014). Recall that these are soft constraints, since we allow for non-compliance with federal and state renewable targets. We also allow for the possibility of trading of Renewable Energy Certificates between states through the combination of constraints (15) and (16), as specified below. Constraint (15) imposes the minimum fraction of the renewable target that has to be met using in-state resources. Constraint (16) ensures that the sum of all state renewable requirements are met using renewable resources within the WECC region. This same formulation was used in Perez et al. (2016).

$$
\begin{array}{ll}
\sum_{h \in H} L_{h} \sum_{i \in G_{R}} y_{i h s}+f u_{s} \geq F R P S \sum_{h \in H} L_{h} \sum_{b \in B} D_{b h s} & \forall s \in S \\
\sum_{h \in H} L_{h} \sum_{i \in G_{R} \cup G_{p}} y_{i h s}+s u_{p s} \geq I N_{p} S R P S_{p} \sum_{h \in H} L_{h} \sum_{b \in B_{p}} D_{b h s} & \forall p \in P, \forall s \in S \\
\sum_{p \in P} \sum_{h \in H} L_{h} \sum_{i \in G_{R} \cup G_{p}} y_{i h s}+s u l b_{s} \geq \sum_{p \in P} S R P S_{p} \sum_{h \in H} L_{h} \sum_{b \in B_{p}} D_{b h s} & \forall s \in S
\end{array}
$$

\footnotetext{
${ }^{7} \mathrm{~A}$ more general formulation would consider the Forced Outage Rates of conventional generators, in which case $W_{i h}$ would be set equal to $\left(1-F O R_{i}\right)$. Such change would not change our basic results.

${ }^{8}$ Our formulation does not account for changes in the reactance of transmission lines due to the investment of new transmission capacity, which is the same simplification used in Sauma and Oren (2006) and Short et al. (2011). This relaxation could bias the location and magnitude of transmission and generation investments (Munoz et al., 2013). However, accounting for changes in the reactance values would result in a non-linear and non-convex model. Solving such model would require application of a customized algorithm, as the one proposed in Ozdemir et al. (2016). The application is beyond the scope of this article since our emphasis on analyzing the impacts of risk aversion.
} 
Generation build limits: These constraints limit investments in new generation capacity depending on the location and type of technology.

$$
x_{i} \leq \bar{X}_{i} \quad \forall i \in G
$$

Transmission build limits: We constrain the amount of capacity that can be added to a transmission corridor in the system. Note that the variable $z_{l}$ is expressed in MW and the parameter $\bar{Z}_{l}$ is expressed as a fraction of a reference transmission line with capacity $T_{l}$.

$$
z_{l} \leq \bar{Z}_{l} T_{l}
$$$$
\forall l \in L
$$

Installed reserves: For the purpose of calculating contributions to installed reserve margins, we derate the capacity of intermittent generation technologies using Effective Load Carrying Capability factors $(E L C C)$ that are based on historical data. ${ }^{9}$

$$
\begin{aligned}
\sum_{i \in G_{N I} \cap G_{j}}\left(X_{i}^{0}+x_{i}\right)+\sum_{i \in G_{I} \cap G_{j}} E L C C_{i}\left(X_{i}^{0}+x_{i}\right) & \\
& \geq\left(1+R M_{j}\right) \sum_{b \in B_{n}} D_{b h^{*} s} \quad \forall j \in J, \forall s \in S
\end{aligned}
$$

Nonnegativity:

$$
x_{i}, z_{l}, y_{i h s}, c_{b h s}, r_{b h s}, f u_{s}, s u_{p s}, a_{s} \geq 0 \quad \forall i \in G, \forall l \in L, \forall h \in H, \forall p \in P, \forall s \in S
$$

\section{Case study: WECC 240-bus system}

\subsection{System description}

We perform our numerical studies using a 240-bus network reduction of the Western Electricity Coordinating Council (WECC). The original dataset was made available by Price and Goodin (2011) and later expanded by Munoz et al. (2014) to perform transmission and generation investment-planning studies. The system has 448 transmission lines and 157 aggregated existing generators. Figure 1 depicts the approximate location of all existing buses and transmission lines in the system.

For transmission upgrades we consider two types of investment alternatives. For existing transmission corridors we allow for the addition of the equivalent

\footnotetext{
${ }^{9}$ This is a frequently-used approach to account for variability of renewable resources in resource adequacy studies. However, it is known that the capacity value of renewable energy technologies - solar in particular-decreases rapidly at increasing penetrations. A method to account for this dependency is proposed in Munoz and Mills (2015). In many markets, the capacity of conventional generators is also derated by expected forced outage rates, but we omit that detail here.
} 

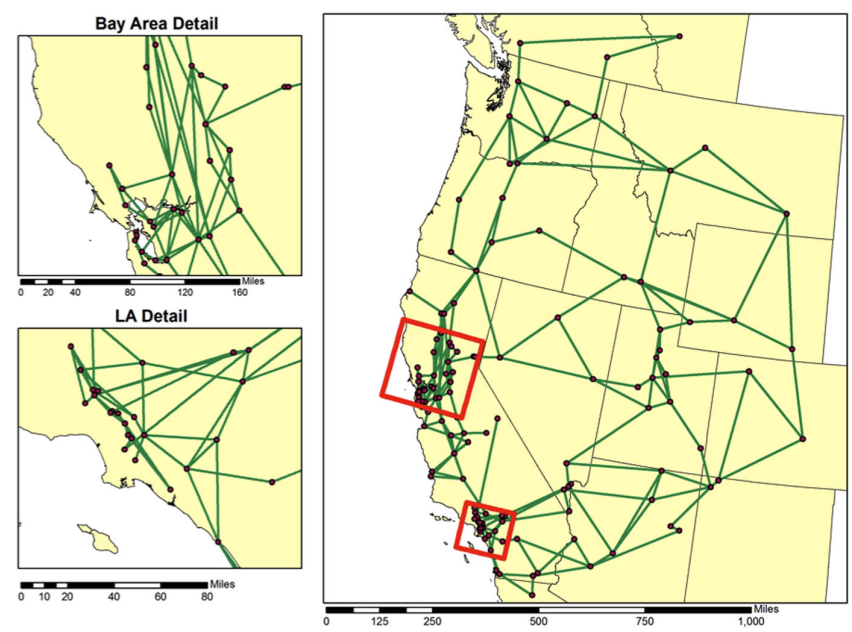

Figure 1: Illustration of the WECC 240-bus system (Munoz et al., 2014).

of up to two new $500 \mathrm{kV}$ circuits and do not consider the possibility of creating new transmission corridors between existing buses. We also allow for the interconnection of renewable hubs to existing buses through radial transmission lines with up to four $500 \mathrm{kV}$ circuits. As implied in the model description in the previous section, we assume that both transmission and generation investments can be made in small increments.

Installed and available generation capacity for new investments are listed in Tables A.6 and A.7, respectively, in the Appendix. We assume that demand can be curtailed at a price of $1000 \$ / \mathrm{MWh}$, which is the price ceiling for most markets in the US. For renewables, including solar, wind, and hydro, we model variability using hourly capacity factors from historical data from the year 2004 . As in Perez et al. (2016), we use 10 representative hours of historical data. A detailed description of the dataset is provided in Munoz (2014), Appendix B.

\subsection{Timing}

In the real world, transmission and generation expansion decisions are made on a rolling basis. The full problem would therefore be a multi-stage or rolling horizon stochastic optimization problem. Due to their size, these problems are difficult to solve even for risk-neutral models with small numbers of scenarios and therefore beyond the scope of this paper. However, we also cannot simply ignore opportunities for recourse: we would significantly overestimate the effects of risk aversion if decision makers were stuck with the effects of their decisions, without any opportunities for further investment, for a long period of time.

We therefore approximate the multi-stage problem by only considering a single decision year, 2034, and annualizing investment costs. This implies that decision makers only incur the costs of their decisions for one year. Of course, we may now understate the results of risk aversion but, since in the real world 
investment decisions are often taken annually, our results will be more representative of a real-world planning situation than those obtained from a model with a much longer planning horizon and no recourse options.

\subsection{Costs}

We assume that the capital cost of all generation technologies for the decade that leads to 2034, which is when investors will commit to the construction of generation capacity for 2034, will be the same as the ones projected by the US Energy Information Administration today (EIA, 2013). Table 1 shows overnight capital cost, fixed O\&M, and net present costs (NPC) for all generation technologies available for investment in the model.

Table 1: Capital costs of generation investment alternatives.

\begin{tabular}{lccccc}
\hline Technology & $\begin{array}{c}\text { Overnight } \\
\text { Capital Cost } \\
(\$ / \mathrm{kW})\end{array}$ & $\begin{array}{c}\text { Fixed } \\
\text { O\&M } \\
(\$ / \mathrm{kW} \text {-year })\end{array}$ & $\begin{array}{c}\text { Lifetime } \\
(\text { years })\end{array}$ & $\begin{array}{c}\mathrm{NPC} \\
(\$ / \mathrm{kW})\end{array}$ & $\begin{array}{c}C X \\
(\$ / \mathrm{kW}-\mathrm{yr})\end{array}$ \\
\hline Coal & 4,579 & 63.21 & 40 & 5,664 & 330.07 \\
CCGT & 978 & 14.39 & 30 & 1,199 & 78.01 \\
CCGTCCS & 2,060 & 30.25 & 30 & 2,525 & 164.26 \\
CT & 665 & 6.7 & 30 & 768 & 49.96 \\
Wind & 2,438 & 28 & 25 & 2,833 & 200.98 \\
Solar & 5,400 & 22 & 25 & 5,710 & 405.14 \\
Biomass & 3,860 & 103 & 30 & 5,443 & 354.10 \\
Geothermal & 4,141 & 84 & 30 & 5,432 & 353.38 \\
Hydro & 3,500 & 15 & 25 & 3,711 & 263.33 \\
\hline
\end{tabular}

The NPC corresponds to the present capital cost of generation, considering both overnight and fixed O\&M costs for the lifetime of each technology, using a discount rate $\delta$ of $5 \%$ per year. Since the planning model optimizes investments for one representative year in the future, we compute the annualized capital cost $C X$ that is then used directly in the optimization model. ${ }^{10}$ For transmission we use the cost estimates detailed in Munoz (2014). To compute the annualized investment costs of transmission $C Z_{l}$, we assume that transmission assets have a lifetime of 50 years and assume the same discount rate $\delta=5 \%$ is used for generation investment alternatives. ${ }^{11}$

We enforce both federal (constraint (14)) and state (constraint (15)) Renewable Portfolio Standards only in the US portion of the WECC, excluding areas of Canada and Mexico. Table 2 shows renewable targets for all states in the

\footnotetext{
${ }^{10} \mathrm{We}$ compute the annualized generation investment cost using the formula $C X=$ $N P C\left(\frac{\delta}{1-\frac{1}{(1+\delta)^{L T}}}\right)$, where $L T$ is the generator lifetime in years.

${ }^{11}$ The capital cost of all transmission investment alternatives are listed in Munoz (2014).
} 
WECC with binding mandates. These are the same projections of RPS targets utilized in Munoz et al. (2014). ${ }^{12}$

Table 2: State-level RPS targets.

\begin{tabular}{lccccccccc}
\hline State & AZ & CA & CO & MT & NM & NV & OR & UT & WA \\
RPS & $15 \%$ & $33 \%$ & $30 \%$ & $15 \%$ & $20 \%$ & $25 \%$ & $13 \%$ & $20 \%$ & $12 \%$ \\
\hline
\end{tabular}

Non-compliance with renewable targets is allowed at a cost of $\$ 50$ per MWh for all states, which is an approximate lower bound for existing fines in the WECC region (Perez et al., 2016). Non-compliance with RPS policies can be also interpreted as the trading of Renewable Energy Certificates between a fringe market and WECC states at a price of $\$ 50$ per MWh, in which case a fraction of the RPS policies within the WECC would be met using eligible renewable resources outside of the WECC region.

\subsection{Scenarios and risk aversion}

Most stochastic transmission and generation planning models use a limited number of scenarios (usually less than 10) for computational reasons. To investigate the effect of risk aversion using CVaRs, this is not enough: as in the real world, we need to consider low-probability/high-consequence scenarios that can have a significant effect on expected profits or welfare if they occur. Moreover, the smallest meaningful CVaR threshold level $\alpha$ in a model with $s$ scenarios is $1 / s$, while typical threshold values are closer to $5 \%$. We therefore define 24 scenarios, which cover a range of possible policy, demand, and cost changes from now until 2034. The scenarios are based on four policy and demand scenarios WECC has recently constructed for use in its long-term planning studies (WECC, 2013). The WECC scenarios describe sets of policies consistent with, respectively, a focus on economic recovery, a focus on clean energy, a focus on short-term consumer costs, and a focus on long-term societal costs, and thus span a broad range of possible futures. Key parameters of these scenarios are summarized in Table 3.

In addition to policy and demand parameters, generation investment is heavily influenced by relative prices of coal and gas, which the WECC scenarios do not fully capture. We therefore define a separate set of three fuel prices scenarios, which are summarized in Table 4 and are broadly in line with predictions made by the International Energy Agency and the US Department of Energy.

\footnotetext{
${ }^{12}$ Note that the projections of renewable mandates used in Munoz et al. (2014) are only approximations of the actual regulations in place. The state of Colorado, for instance, imposes different renewable targets for investor-owned utilities (30\%) and municipalities (10\%). For all states with RPS mandates such as the one in Colorado we use the maximum enforced target, assuming that climate change concerns will actually drive renewable targets towards more stringent levels than the ones considered today. Perez et al. (2016) proposes a deterministic planning model with more realistic features than the ones we consider in this article, including explicit modeling of the geographical eligibility and trading of Renewable Energy Certificates between states. However, replicating RPS policies in the WECC with such level of detail is
} 


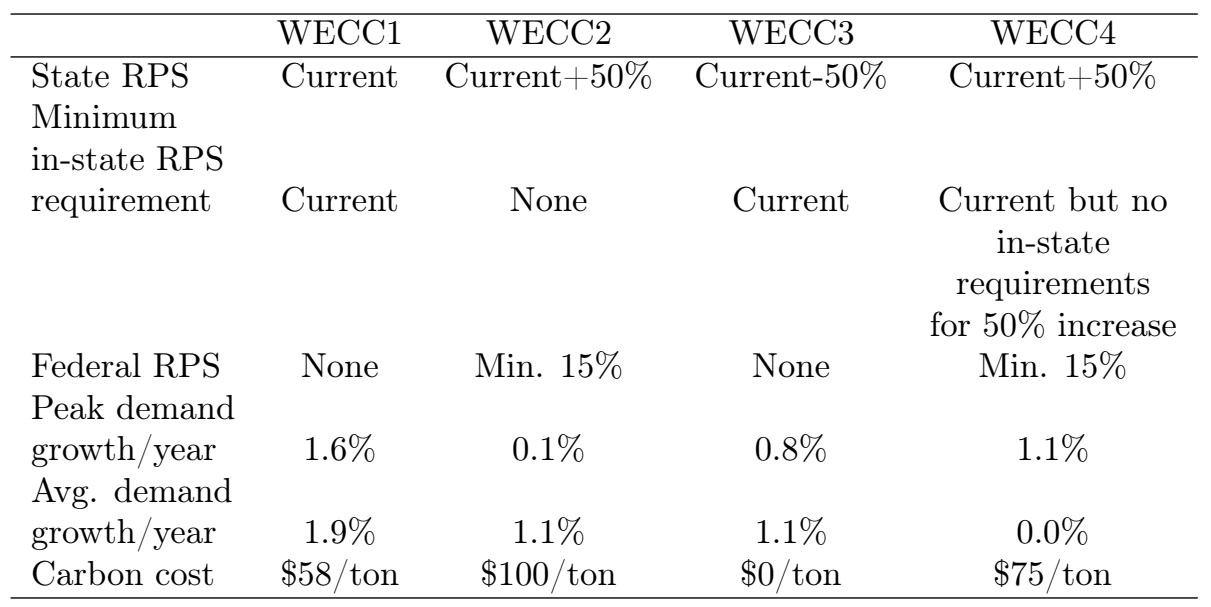

Table 3: Policy/demand scenarios

\begin{tabular}{lccc}
\hline & Cheaper gas & Cheaper coal & Current levels \\
\hline Gas price & $\$ 2 / \mathrm{MMBtu}$ & $\$ 10 / \mathrm{MMBtu}$ & $\$ 5 / \mathrm{MMBtu}$ \\
Coal price & $\$ 2.50 / \mathrm{MMBtu}$ & $\$ 1.50 / \mathrm{MMBtu}$ & $\$ 2 / \mathrm{MMBtu}$ \\
\hline
\end{tabular}

Table 4: Fuel price scenarios

A third important determinant of generation investment is the availability of nuclear capacity, which, as history has shown, can change rapidly in response to nuclear accidents; a third set of two scenarios, which are summarized in Table 5, captures these possibilities. The $50 \%$ retirement of nuclear capacity corresponds roughly to the amount of nuclear generation capacity in WECC constructed before 1990, which s also similar to the amount of nuclear capacity retired by Germany in the aftermath of the Fukushima disaster; it is therefore our best estimate of the magnitude of a government response to a nuclear accident.

To construct our final set of 24 scenarios, we simply take the Cartesian product of the four WECC policy/demand scenarios, the three fuel price scenarios, and the two nuclear scenarios, in the absence of any evidence to support correlations between the probabilities of scenarios in each set. For simplicity, we assume that each of the 24 scenarios is equiprobable, although of course other assumptions could be made. We will initially use a CVaR threshold of

beyond the scope of this article.

\begin{tabular}{lcc}
\hline & Status quo & Nuclear accident \\
\hline Nuclear capacity & Current levels & Current levels $-50 \%$ \\
\hline
\end{tabular}

Table 5: Nuclear scenarios 


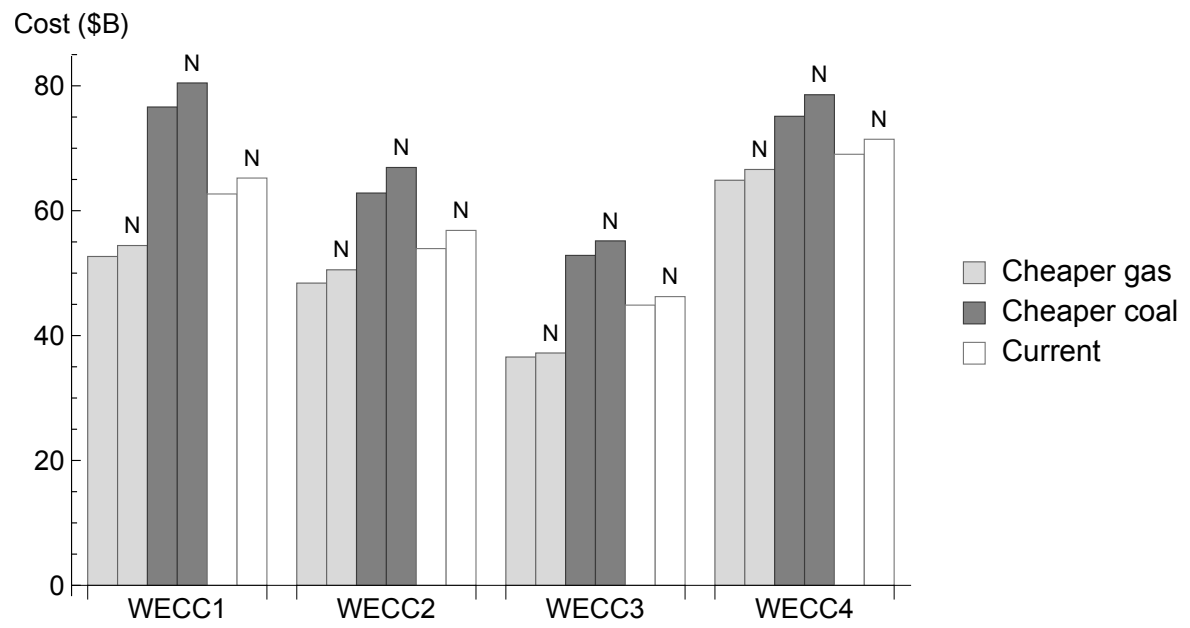

Figure 2: Costs in individually optimized scenarios

$\alpha=0.916$, such that the CVaR selects the two highest-cost scenarios, and vary the weight on the $\mathrm{CVaR}, \omega$, from 0 (risk neutrality) to 1 (extreme risk aversion). In section 5.4, we explore the effect of changing $\alpha$.

Figure 2 shows the system costs for individually transmission-optimized scenarios, i.e., the system costs if one scenario is known to occur with probability 1. The bars marked "N" are the scenarios in which nuclear capacity is reduced. As this figure shows, there is significant variation in system costs between the scenarios; as may be expected, scenarios with higher RPSs, lower coal rather than lower gas prices, and less nuclear capacity are more costly. That does not necessarily imply that these scenarios are the most risky in a stochastic planning framework - although they are likely to be - but it does show that some scenarios are inherently more costly, even if they are anticipated.

\section{Results}

\subsection{Investment, costs and risk}

Figures 3 and 4 show how investment in transmission (backbones and interconnections to renewable hubs) and generation change with $\omega$, the weight on the CVaR of the tail of the cost distribution in the objective function. A higher $\omega$ implies a higher weight on the more costly scenarios, and hence, a higher level of risk aversion; when $\omega=0$, investors are risk-neutral. As these figures show, the impact of risk aversion on these aggregate transmission and generation investment levels in the WECC is minor. Investment in generation increases slightly with risk aversion, as does investment in interconnection capacity to renewable hubs. Investment in transmission backbones decreases slightly with moderate levels of risk aversion, since it is a substitute for generation in some locations, and increases again for extreme risk averse cases to accommodate more 


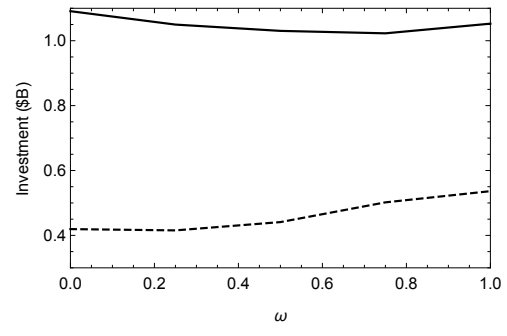

- Backbones ------ Interconnections

Figure 3: Transmission investment

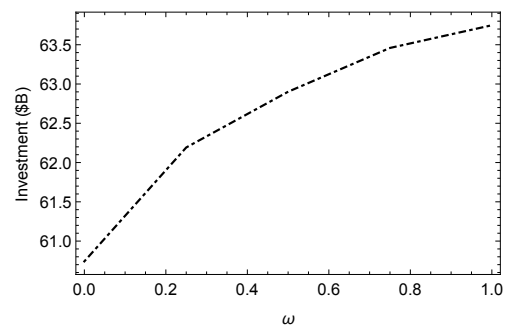

Figure 4: Generation investment

renewable investment in different locations. The total amount of transmission investment increases slightly with risk aversion.

We will discuss the reasons for these changes in more detail when we look at the spatial distributions of investment, but before we do so, it is worth exploring why risk aversion seems to have such a limited effect on overall levels of generation and transmission investment. To do so, Fig. 5 shows how the cumulative probability density function of costs changes if we move from a risk-neutral case $(\omega=0)$ to a moderately risk-averse case $(\omega=1 / 2)$, and a case in which only costs in the worst few scenarios enter the objective function $(\omega=1)$. This suggests several reasons for the limited effect of risk aversion. First, a small change in the patterns of investment already significantly reduces the costs in the worst few scenarios, suggesting that the objective function is relatively flat around its optimum. There are relatively cheap actions that can be taken to reduce exposure to risk (e.g., investment in renewable capacity, which in some locations is already close to competitive in a risk-neutral model); naturally, these increase costs in low-cost scenarios, but the trade-off is not severe. Secondly, once these actions are taken, there is little that can be done to further reduce risk. Even if investors only consider the worst scenarios, costs in those scenarios cannot be reduced further. In Fig. 5, these worst scenarios are scenarios with high RPS levels, high demand growth, and high fuel prices. Even if investors had certainty that one of these would occur, there is no 'get out of jail free'-card - costs can be reduced somewhat, but these futures are still expensive, as Fig. 2 already indicated. These two reasons also explain why, as Fig. 5 shows, the level of risk 


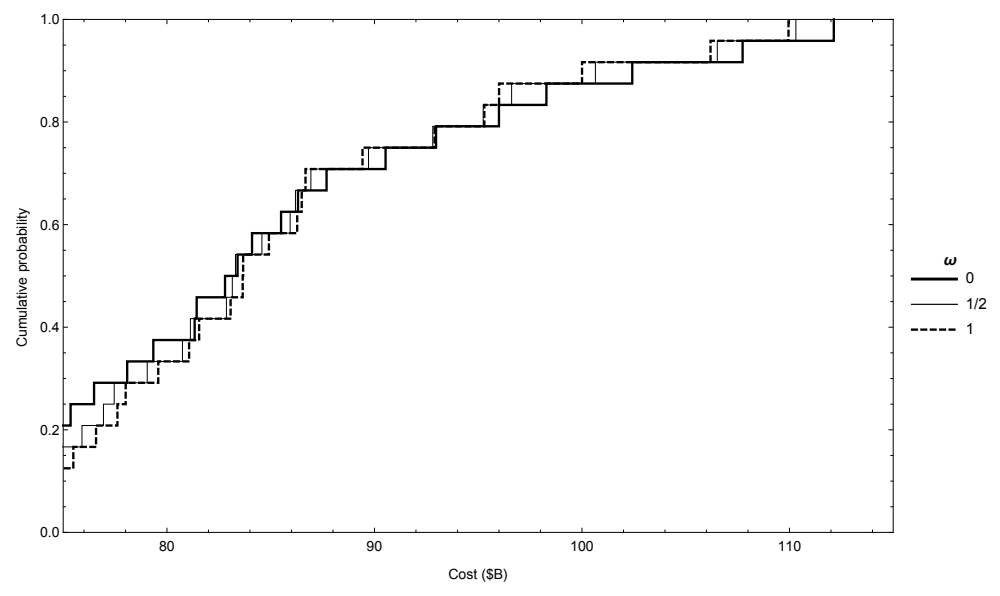

Figure 5: CDFs of costs

aversion seems to matter little once we move to a case with $\omega>0$.

The existing literature suggests a third reason for the relative insensitivity of total investment levels to risk aversion. In risk-neutral stochastic planning models, it has been shown that the number of scenarios is of relative unimportance, while the range of scenarios is an important driver of model outcomes (Hobbs et al., 2015). This suggests that the most extreme scenarios drive much of the results of a risk-neutral model, and hence, putting an even higher weight on them in a risk-averse model does not change the results significantly.

\subsection{Generation investment}

Figure 7 shows how investment in the various different types of generation capacity changes as a result of an increasing weight $\omega$ on costs in the worst scenarios, relative to the risk-neutral solution (see Figure B.10 in the Appendix for more details on the risk-neutral solution). There are no appreciable changes in hydro and coal investment, both of which are low in all scenarios because of limited opportunities of expansion and environmental constraints. As this figure shows, in our model higher, levels of risk aversion lead to more investment in low-carbon technologies, particularly wind and solar. Investment in the most carbon-intensive technology, open-cycle gas turbines $(\mathrm{CT})$, decreases significantly with risk aversion.

As mentioned above, the highest-cost scenarios are those with high state and federal RPSs and high fuel prices. It is therefore not surprising that the best hedge against those scenarios involves investing in renewables, which helps to meet RPS targets and, once constructed, their variable costs do not depend on fuel prices. This effect is not universal. In markets where there are current high levels of support for renewables through feed-in tariffs or other mechanisms which directly effect the marginal profits of generators, and high probabilities that these support mechanisms are stopped, renewables may well be riskier, 


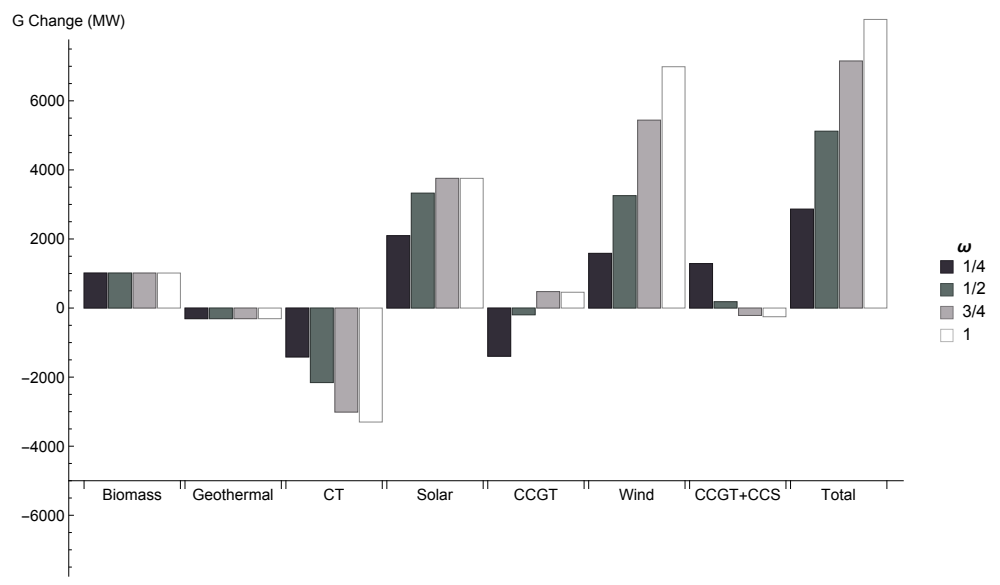

Figure 6: Generation investment changes by type relative to the risk neutral solution.

and investment would decrease with risk aversion. In addition, short-term uncertainty could increase risks related to renewable developments (Seljom and Tomasgard, 2015). In our setting, with our scenarios, however, they help reduce policy and fuel cost risk. Indeed, there is anecdotal evidence that US investors are increasingly looking at renewables for these purposes (Haemig, 2015).

In general, it is notable that the differences in investment levels for CTs and wind between risk-neutral and risk-averse models is much higher than the difference in total investment levels. Thus, even if risk aversion has a minimal effect on total investments or costs, there are still significant differences in the generation mix.

A similar, even more striking effect can be observed in the spatial patterns of investment. Figure 7 shows how the spatial distribution of generation investment changes as a result of an increasing weight $\omega$ on costs in the worst scenarios, relative to expected costs. Again, the differences between risk-neutral and risk-averse results are much bigger in some individual states, such as Arizona, than the net investment differences, and certainly large enough to be of major interest to local policy makers and planners. The spatial patterns are mostly a result of the presence of good renewable resources, existing transmission capacity, and renewable policies. The largest differences can be observed in Arizona, which has excellent renewable resources and is well-connected to California and other coastal states with relatively high RPSs, and where risk aversion leads to a significant increase in generation investment. In neighboring New Mexico, on the other hand, investment decreases, because it is less well connected and can now import generation from Arizona. Generation investment in California is relatively independent of risk aversion compared to Arizona, New Mexico, and Wyoming, partly because it benefits from the increased investment in Arizona and because RPSs are already high at present, leading to large amounts of investment in any scenario and thus less exposure to policy risk. 


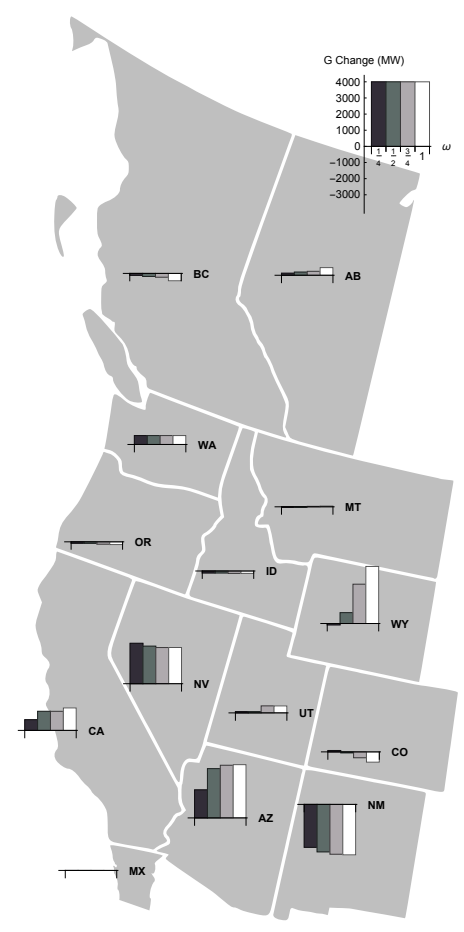

Figure 7: Generation investment changes by location relative to the risk neutral solution.

A final observation from both these figures is that the effects of risk aversion are often non-monotonic, likely because of the complex interplay between the various renewable constraints and between generation and transmission investments. Besides, cost functions for generation and transmission are linear, which implies that a small change in the model input parameters can lead to a large change in the primal solution variables. This implies that these interactions need to be modeled carefully before policy is designed, and that they cannot be considered in isolation.

\subsection{Transmission investment}

Figure 8 shows how the spatial distribution of transmission investment changes as a result of an increasing weight $\omega$ on costs in the worst scenarios, relative to the risk-neutral solution (see Figure B.11 in the Appendix for more details on the risk-neutral solution). As we have already seen above, the total amount of transmission capacity increases slightly with risk aversion; as with generation, the differences in individual states are much larger. In some states, such as Arizona, transmission is clearly a substitute for generation, and because of the sharp increase in generation investment in the risk averse case, the transmission planner can reduce its investment there. In other states such as Wyoming, 
transmission investment to renewable hubs increases, anticipating more investment in renewable generation capacity. Existing capacities play an important role here, too; transmission investment in California is noticeably higher under risk-averse than under the risk-neutral assumptions, even though generation investment does not change much, such that surplus of renewable power can be exported to other states in scenarios without in-state RPS requirements.

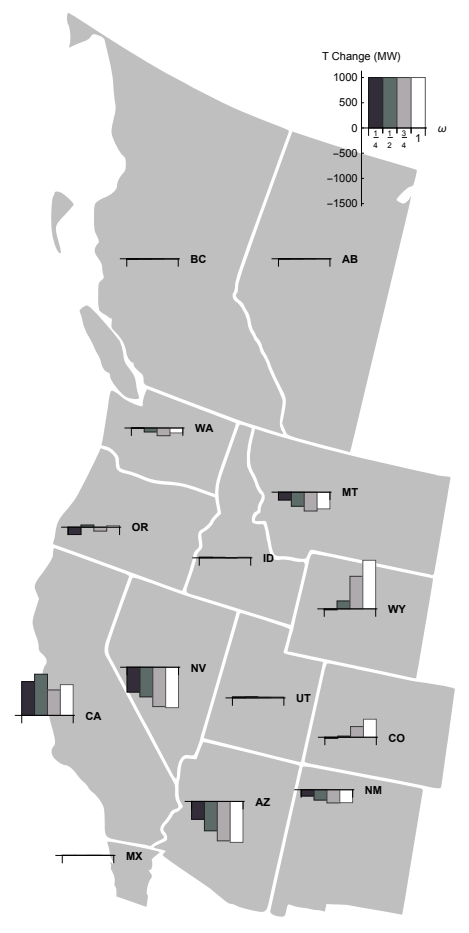

Figure 8: Transmission investment changes by location relative to the risk neutral solution.

The effects of risk aversion on transmission investment are therefore very different from state to state. As with generation investment, they are also highly non-monotonic, making prediction difficult.

\subsection{Sensitivity to tail definition}

Throughout the above, we have set the CVaR threshold $\alpha=0.916$ such that, in the model objective, the two endogenously selected highest-cost scenarios received a higher weight. This threshold value is in line with what is typically used in most research studies; however, for a better understanding of how far our results are driven by a limited number of scenarios, here we present results from a sensitivity analysis with respect to this parameter. Figure 9 shows changes in two selected technologies, CT and wind, as well as changes in generation and transmission investment costs with respect to the risk-neutral solution for different values of $\alpha$ and $\omega$. 
We find that most of our conclusions regarding the effect risk aversion on generation and transmission investments remain valid for larger values of $\alpha$. Although absolute levels of investments in generation per technology change as we increase $\alpha$, the results show that increasing risk aversion (i.e., giving more weight to the CVaR by increasing $\omega$ and less to the expected system cost in the objective function) leads to more investments in wind capacity and less in CT for all the values of $\alpha$ shown in the figure. Similarly, more risk aversion leads to higher investment costs in aggregate generation capacity and in transmission infrastructure. This is consistent with our analysis in Section 5.2, since the scenarios with the highest costs are those with high renewable requirements. Generation and transmission investment by state show small differences. As expected, including a larger number of scenarios on the CVaR mostly reduces the difference between a risk-neutral and risk-averse, although the changes are non-monotonic. For smaller values of $\omega$, the differences are even smaller.
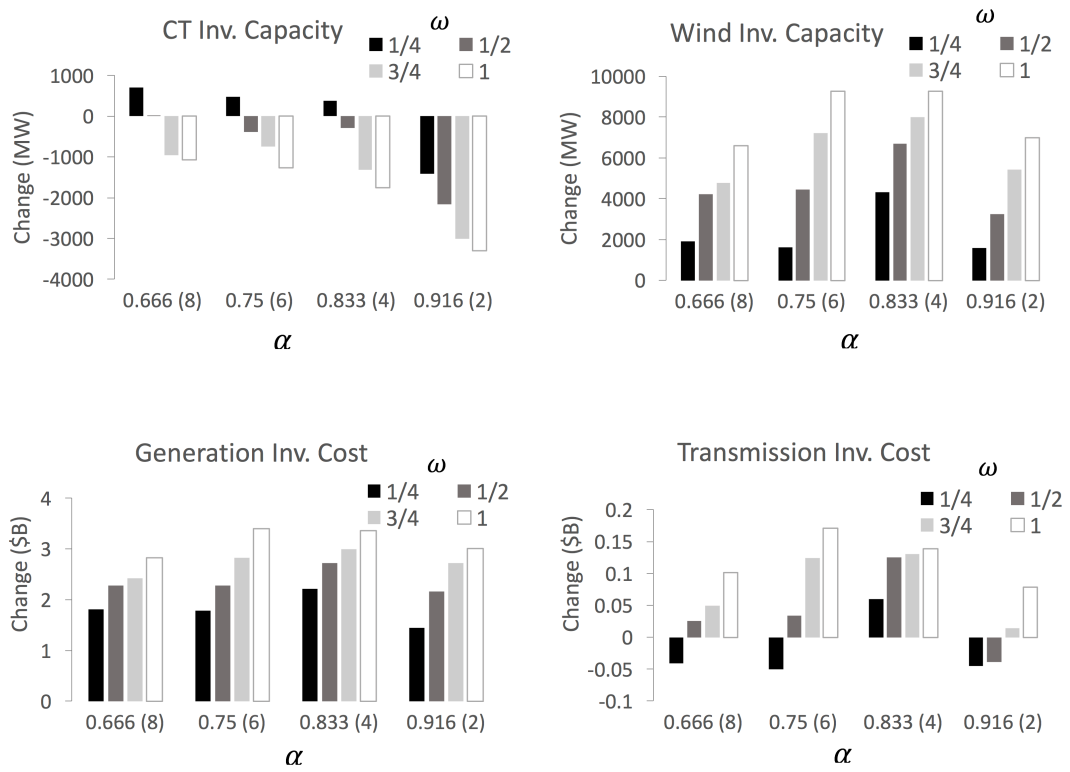

Figure 9: Changes in $\mathrm{CT}$ and wind investments as well as in generation and transmission investment costs with respect to the risk-neutral solution for different values of the confidence level $\alpha$. The numbers in parentheses indicate the number of scenarios included in the CVaR calculation for each $\alpha$. 


\section{Conclusions}

In this paper, we have investigated the effects of risk aversion on electricity generation and transmission planning in a large network. Hitherto, most studies focused on either transmission or generation planning; as we have shown above, there are important interactions between the effects of risk aversion on both of these, so a combined approach is clearly needed. Most existing studies also focus on small test cases. However, these cannot be used to fully capture the spatial differences in investment between risk-neutral and risk-averse models which, based on our results, can also be relevant. We have shown how a risk-averse Stackelberg transmission-generation expansion equilibrium is equivalent to a risk-averse cost minimization under some reasonable assumptions, and applied this model to a 240-bus representation of the WECC network.

Our results indicate that, at least for our parametrization, risk aversion has only a small impact on overall levels of transmission and generation investment, because minor adjustments to investment patterns already reduce risk significantly, thus limiting further hedging possibilities. In addition, extreme scenarios already drive much of the results of a risk-neutral model. Although in our model overall investment levels do not change much, there are significant regional impacts, mainly because of a shift from carbon-intensive to carbon-neutral generation and a changed transmission investment pattern that anticipates these developments in new generation capacity. This highlights the importance of considering risk aversion in planning models, especially if these are used for policy making. Importantly, the effect of risk aversion on investment in particular technologies or in particular places is often non-monotonic because of the complex interactions between renewable policies and between generation and transmission investment. This implies that they will be hard to predict and that they are sensitive to particular assumptions about investors' attitudes to risk and other constraints, such as renewable targets.

Naturally, our model is still simplified. Because we assume a perfectly competitive generation market and a complete financial market, our model misses some of the strategic interaction between investors that occur in the real world. We may also overestimate investment as a result of not taking into account that financial markets are, in reality, incomplete. Moreover, we only consider a onestage game with annualized investment costs, which implies that investors only live with the results of bad decisions for one year, which could well underestimate the magnitude of a real regret. We also assume that all generators and the transmission planner have the same attitude to risk. Further research into the application of multi-level equilibrium models to risk-averse energy investment applications is necessary to investigate the impact of these simplifications. Nevertheless, our results are consistent with theory and anecdotal evidence, and we expect the same effects that we find to carry over to models with more detailed market representations or fine-grained time dimensions. 


\section{Acknowledgements}

The research in this article was supported by FONDECYT \#11150029, CONICYT/FONDAP/15110019 (SERC-CHILE), Basal Project FB0008, the UK Engineering and Physical Sciences Research Council through grant number EP/P001173/1 (CESI) and the U.S. Department of Energy's Office of Science through the Advanced Scientific Computing Research program, under contract number KJ0401000, project title "Multifaceted Mathematics for Complex Energy Systems." Sandia National Laboratories is a multi-program laboratory managed and operated by Sandia Corporation, a wholly owned subsidiary of Lockheed Martin Corporation, for the U.S. Department of Energy's National Nuclear Security Administration under Contract DE-AC04-94-AL85000.

\section{References}

Alguacil, N., Arroyo, J. M., Carrión, M., 2010. Transmission network expansion planning under deliberate outages. In: Rebennack, S., Pardalos, P. M., Pereira, M. V. F., Iliadis, N. A. (Eds.), Handbook of Power Systems I. Springer-Verlag, Berlin.

Arroyo, J. M., Alguacil, N., Carrión, M., 2010. A risk-based approach for transmission network expansion planning under deliberate outages. IEEE Transactions on Power Systems 25 (3), 1759-1766.

Barradale, M. J., 2010. Impact of public policy uncertainty on renewable energy investment: Wind power and the production tax credit. Energy Policy 38 (12), 7698-7709.

Bellman, R., 1952. On the theory of dynamic programming. Proceedings of the National Academy of Sciences 38 (8), 716-719.

Ben-Tal, A., Nemirovski, A., 2002. Robust optimization-methodology and applications. Mathematical Programming 92 (3), 453-480.

Chen, B., Wang, J., Wang, L., He, Y., Wang, Z., 2014. Robust optimization for transmission expansion planning: Minimax cost vs. minimax regret. IEEE Transactions on Power Systems 29 (6), 3069-3077.

De la Torre, T., Feltes, J. W., Román, T. G. S., Merrill, H. M., 1999. Deregulation, privatization and competition: transmission planning under uncertainty. IEEE Transactions on Power Systems 14 (2), 460-465.

Delgado, D., Claro, J., 2013. Transmission network expansion planning under demand uncertainty and risk aversion. Electrical Power and Energy Systems 44 (1), 696-702.

Deng, S., Oren, S., 2006. Electricity derivatives and risk management. Energy 31 (67), 940-953. 
Duffie, D., Pan, J., 1997. An overview of value at risk. The Journal of derivatives $4(3), 7-49$.

Eeckhoudt, L., Gollier, C., Schlesinger, H., 2005. Economic and financial decisions under risk. Princeton University Press.

Ehrenmann, A., Smeers, Y., 2011a. Generation capacity expansion in a risky environment: A stochastic equilibrium analysis. Operations Research 59 (6), $1332-1346$.

Ehrenmann, A., Smeers, Y., 2011b. Stochastic equilibrium models for generation capacity expansion. In: Bertochhi, M., Consigli, G., Dempster, M. A. H. (Eds.), Stochastic optimization methods in finance and energy. No. 163 in International Series in Operations Research \& Management Science. Springer Science+Business Media LLC, Ch. 13, pp. 273-310.

EIA, 2013. Updated Capital Cost Estimates for Utility Scale Electricity Generating Plants. US Energy Information Administration. http://www.eia.gov/ forecasts/capitalcost/pdf/updated_capcost.pdf.

Fan, L., Hobbs, B. F., Norman, C. S., 2010. Risk aversion and $\mathrm{CO}_{2}$ regulatory uncertainty in power generation investment: policy and modeling implications. Journal of Environmental Economics and Management 60 (3), 193-208.

Fishburn, P. C., 1970. Utility theory for decision making. Tech. rep., DTIC Document.

Fuss, S., Szolgayova, J., Obersteiner, M., Gusti, M., 2008. Investment under market and climate policy uncertainty. Applied Energy 85 (8), 708-721.

Go, R. S., Munoz, F. D., Watson, J.-P., 2016. Assessing the economic value of cooptimized grid-scale energy storage investments in supporting high renewable portfolio standards. Applied Energy 183, 902-913.

Haemig, K., 2015. Precautionary hedging of local power markets using renewables. In: Transatlatic Infraday Conference. Washington, DC.

Hobbs, B. F., Ho, J., Spyrous, E., Donohoo, P., Xu, Q., Ouyang, J., Johnson, R., Ozdemir, O., July 2015. Which oversimplification is the worst sin? lessons from large scale transmission planning for renewables using optimization in the Eastern and Western interconnections of the US. In: wholeSEM Annual Conference.

Hu, M.-C., Hobbs, B. F., 2010. Analysis of multi-pollutant policies for the us power sector under technology and policy uncertainty using markal. Energy 35 (12), 5430-5442.

Huang, Y.-H., Wu, J.-H., 2008. A portfolio risk analysis on electricity supply planning. Energy Policy 36 (2), $627-641$. 
Hugonnier, J., Morellec, E., 2007. Corporate control and real investment in incomplete markets. Journal of Economic Dynamics \& Control 31 (5), 17811800 .

Jabr, R. A., 2013. Robust transmission network expansion planning with uncertain renewable generation and loads. IEEE Transactions on Power Systems 28 (4), 4558-4567.

Jin, S., Ryan, S. M., Watson, J.-P., 2011. Modeling and solving a large-scale generation expansion planning problem under uncertainty. Energy Systems $2(3-4), 209-242$.

Kamalinia, S., Shahidehpour, M., Wu, L., 2014. Sustainable resource planning in energy markets. Applied Energy 133, 112-120.

Kettunen, J., Bunn, D. W., Blyth, W., 2011. Investment propensities under carbon policy uncertainty. The Energy Journal 32 (1), 77-118.

Krishnan, V., Ho, J., Hobbs, B. F., Liu, A. L., McCalley, J. D., Shahidehpour, M., Zheng, Q. P., 2015. Co-optimization of electricity transmission and generation resources for planning and policy analysis: review of concepts and modeling approaches. Energy Systems, 1-36.

López, J. Á., Ponnambalam, K., Quintana, V. H., 2007. Generation and transmission expansion under risk using stochastic programming. IEEE Transactions on Power Systems 22 (3), 1369-1378.

Merrill, H. M., Wood, A. J., 1991. Risk and uncertainty in power system planning. International Journal of Electrical Power \& Energy Systems 13 (2), 81-90.

Meunier, G., 2013. Risk aversion and technology mix in an electricity market. Energy Economics 40, 866-874.

Moreira, A., Street, A., Arroyo, J. M., 2015. An adjustable robust optimization approach for contingency-constrained transmission expansion planning. IEEE Transactions on Power Systems 30 (4), 2013-2022.

Mulvey, J. M., Vanderbei, R. J., Zenios, S. A., 1995. Robust optimization of large-scale systems. Operations Research 43 (2), 264-281.

Munoz, F. D., 2014. Engineering-Economic Methods for Power Transmission Planning under Uncertainty and Renewable Resource Policies. Ph.D. thesis, The Johns Hopkins University.

Munoz, F. D., Hobbs, B. F., Ho, J. L., Kasina, S., 2014. An engineeringeconomic approach to transmission planning under market and regulatory uncertainties: WECC case study. IEEE Transactions on Power Systems 29 (1), $307-317$. 
Munoz, F. D., Mills, A. D., Oct 2015. Endogenous assessment of the capacity value of solar pv in generation investment planning studies. IEEE Transactions on Sustainable Energy 6 (4), 1574-1585.

Munoz, F. D., Sauma, E. E., Hobbs, B. F., 2013. Approximations in power transmission planning: implications for the cost and performance of renewable portfolio standards. Journal of Regulatory Economics 43 (3), 305-338.

Munoz, F. D., Watson, J.-P., Hobbs, B. F., 2015. Optimizing your options: Extracting the full economic value of transmission when planning under uncertainty. The Electricity Journal 28 (5), 26-38.

Neuhoff, K., de Vries, L., 2004. Insufficient incentives for investment in electricity generation. Utilities Policy 12 (4), 253-267.

Ozdemir, O., Munoz, F. D., Ho, J. L., Hobbs, B. F., March 2016. Economic analysis of transmission expansion planning with price-responsive demand and quadratic losses by successive lp. IEEE Transactions on Power Systems 31 (2), 1096-1107.

Perez, A. P., Sauma, E. E., Munoz, F. D., Hobbs, B. F., 2016. The Economic Effects of Interregional Trading of Renewable Energy Certificates in the U.S. WECC. The Energy Journal 37 (4).

Pisciella, P., Vespucci, M. T., Bertocchi, M., Zigrino, S., 2014. A time consistent risk averse three-stage stochastic mixed integer optimization model for power generation capacity expansion. Energy Economics 53, 203 - 211.

Pozo, D., Contreras, J., Sauma, E., 2013a. If you build it, he will come: Anticipative power transmission planning. Energy Economics 36, 135-146.

Pozo, D., Sauma, E. E., Contreras, J., 2013b. A three-level static milp model for generation and transmission expansion planning. IEEE Transactions on Power Systems 28 (1), 202-210.

Price, J. E., Goodin, J., 2011. Reduced network modeling of wecc as a market design prototype. In: IEEE Power and Energy Society General Meeting. pp. $1-6$.

Ralph, D., Smeers, Y., 2010. The invisible hand for risk averse investment in electricity generation. In: Chen, B. (Ed.), Proceedings, 6th International Conference on Algorithmic Aspects in Information and Management. p. 12.

Rockafellar, R. T., Uryasev, S., 2000. Optimization of conditional value-at-risk. Journal of risk 2, 21-42.

Roh, J. H., Shahidehpour, M., Wu, L., 2009. Market-based generation and transmission planning with uncertainties. IEEE Transactions on Power S 24 (3), $1587-1598$. 
Roques, F., Hiroux, C., Saguan, M., 2010. Optimal wind power deployment in europe - a portfolio approach. Energy Policy 38 (7), 3245-3256.

Roques, F. A., Newbery, D. M., Nuttall, W. J., 2008. Fuel mix diversification incentives in liberalized electricity markets: a mean-variance portfolio theory approach. Energy Economics 30 (4), 1831-1849.

Ruiz, C., Conejo, A. J., 2015. Robust transmission expansion planning. European Journal of Operational Research 242 (2), 390-401.

Sardinha, H. R., Sousa, J. M. C., Silva, C. A., Delgado, D., Claro, J., 2013. Transmission network expansion planning under uncertainty using the conditional value at risk and genetic algorithms. In: Proceedings, INTELLI 2013: The Second International Conference on Intelligent Systems and Applications. pp. 135-140.

Sauma, E. E., Oren, S. S., 2006. Proactive planning and valuation of transmission investments in restructured electricity markets. Journal of Regulatory Economics 30 (3), 261-290.

Seljom, P., Tomasgard, A., 2015. Short-term uncertainty in long-term energy system models - a case study of wind power in Denmark. Energy Economics 49, 157-167.

Short, W., Sullivan, P., Mai, T., Mowers, M., Uriarte, C., Blair, N., Heimiller, D., Martinez, A., 2011. Regional Energy Deployment System (ReEDS). Tech. Rep. NREL/TP-6A20-46534, National Renewable Energy Laboratory.

van der Weijde, A. H., Hobbs, B. F., 2012. The economics of planning electricity transmission to accommodate renewables: Using two-stage optimisation to evaluate flexibility and the cost of disregarding uncertainty. Energy Economics 34 (6), 2089-2101.

WECC, 2013. WECC Long-Term Planning Scenario Report. URL https://www. wecc.biz/TEPPC/Pages/SPSG . aspx

Willems, B., Morbee, J., 2010. Market completeness: How options affect hedging and investments in the electricity sector. Energy Economics 32 (4), 786-795.

Zheng, Q. P., Pardalos, P. M., 2010. Stochastic and risk management models and solution algorithm for natural gas transmission network expansion and LNG terminal location planning. Journal of Optimization Theory and Applications 147 (2), 337-357. 
Appendix A. Generation information 


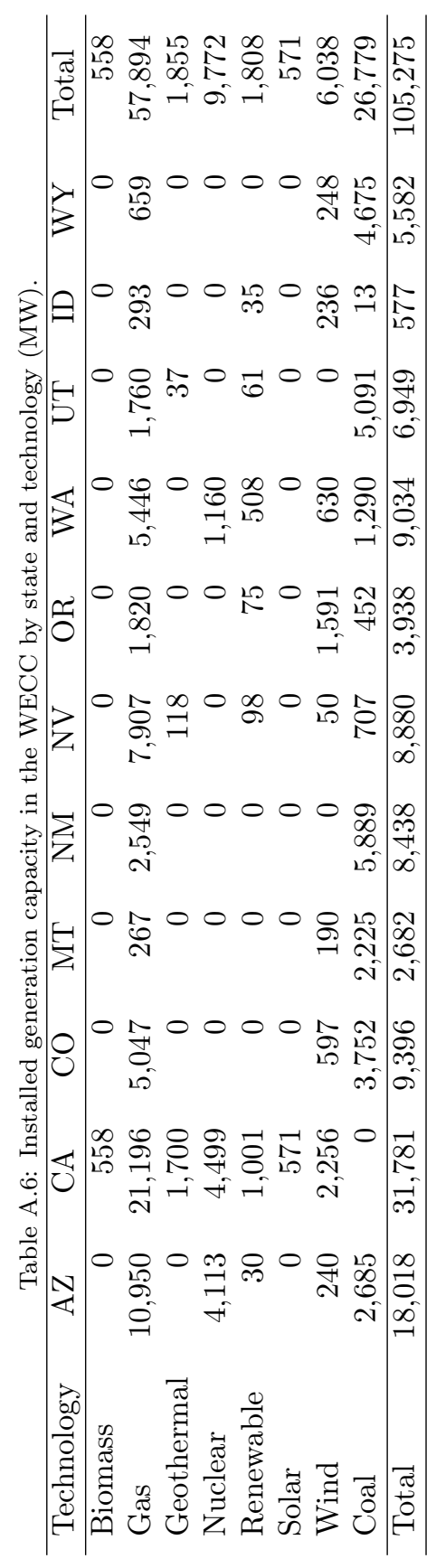




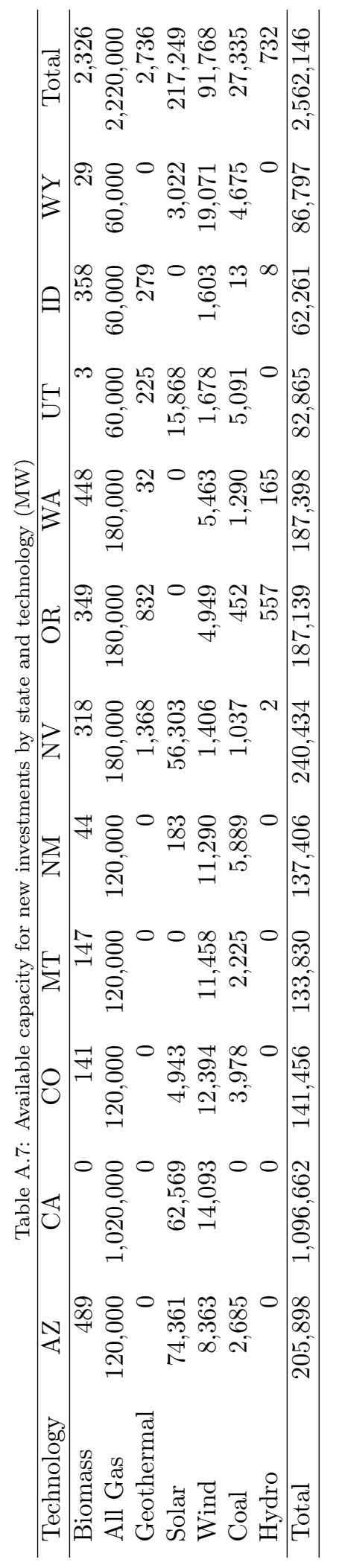




\section{Appendix B. Risk-neutral results}

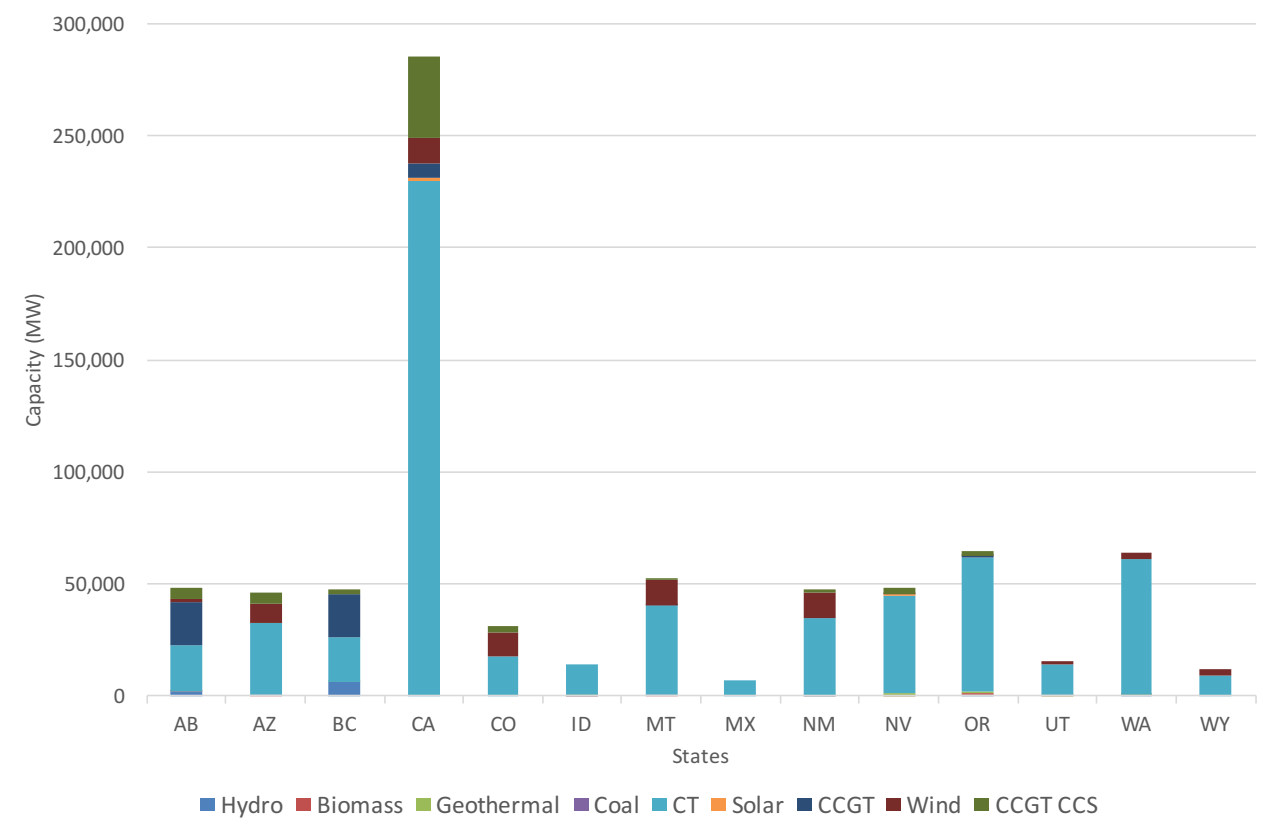

Figure B.10: Generation investments per state and technology for the risk-neutral solution.

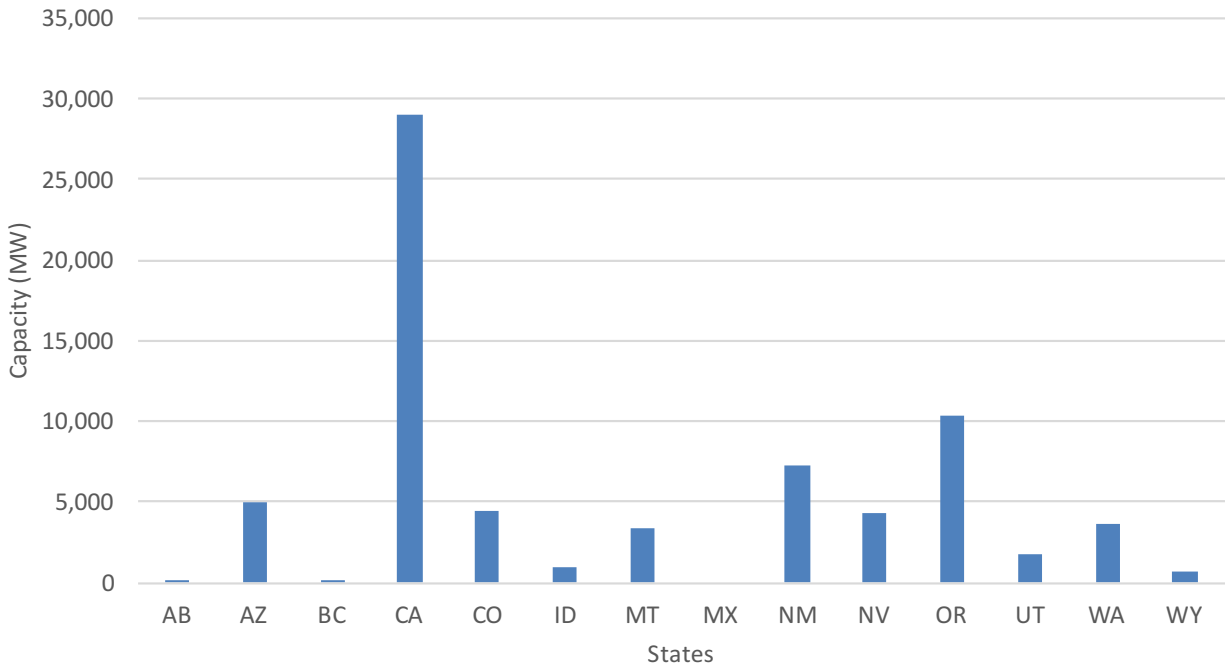

Figure B.11: Transmission investments per state for the risk-neutral solution. 